\title{
Electromagnetic Field Imaging in Arbitrary Scattering Environments
}

\author{
Karteekeya Sastry ${ }^{\circledR}$, Chandan Bhat ${ }^{\circledR}$, Raffaele Solimene ${ }^{\circledR}$, Senior Member, IEEE, and Uday K. \\ Khankhoje ${ }^{\circledR}$, Senior Member, IEEE
}

\begin{abstract}
In this article, we propose a method to reconstruct the total electromagnetic field in an arbitrary two-dimensional scattering environment without any prior knowledge of the incident field or the permittivities of the scatterers. However, we assume that the region between the scatterers is homogeneous and that the approximate geometry describing the environment is known. Our approach uses field measurements and a compressive sensing inspired algorithm to estimate the incident field and the tangential electric and magnetic fields on the scatterers' surfaces. These estimates are then used to predict the field everywhere using Huygens' principle. Further, we identify the best measurement locations in the environment, which reduces the estimation error to approximately half of the error obtained when using random locations. We show that in an indoor scenario with up to four scattering objects, the total electric field is recovered with less than $10 \%$ error when the number of measurements is just 0.3 times the number of unknowns in which the problem is formulated. The formulated problem is solved using 'Total field - Compressive sensing based subspace optimization method' - an algorithm that leverages the sparsity of the tangential fields in known transformation domains to obtain an optimal solution.
\end{abstract}

Index Terms-Compressive sensing, electromagnetic fields, sensor placement, subspace optimization.

\section{INTRODUCTION}

$\mathbf{K}$ NOWLEDGE of the electromagnetic (EM) fields is useful in many applications like network planning, $\mathrm{WiFi}$ access point planning in indoor scenarios, and indoor localization [1][4]. Existing methods in the literature that address this problem are based on ray tracing [5], [6]. It is well known that the ray-tracing model fails to account for the effects of diffraction

Manuscript received August 22, 2020; revised November 29, 2020; accepted January 27, 2021. Date of publication February 1, 2021; date of current version February 19, 2021. This work was supported by the Microwave Inverse Scattering for Breast Cancer Detection project through the Science and Engineering Research Board, Department of Science and Technology, Government of India, under Grant ECR/2018/001953. The associate editor coordinating the review of this manuscript and approving it for publication was Dr. Ilaria Catapano. (Karteekeya Sastry and Chandan Bhat contributed equally to this work.) (Corresponding authors: Chandan Bhat; Uday K. Khankhoje.)

Karteekeya Sastry is with the Department of Electrical Engineering, California Institute of Technology, Pasadena, CA 91125 USA (e-mail: karteekdhara98@gmail.com).

Chandan Bhat and Uday K. Khankhoje are with the Department of Electrical Engineering, Indian Institute of Technology Madras, Chennai 600036, India (e-mail: chandanbhat21@gmail.com; uday@ee.iitm.ac.in).

Raffaele Solimene is with the Universita della Campania Luigi Vanvitelli, Italy, and is also an Adjunct Faculty with the Department of Electrical Engineering, Indian Institute of Technology Madras, Chennai 600036, India (e-mail: raffaele.solimene@unicampania.it).

Digital Object Identifier 10.1109/TCI.2021.3055982

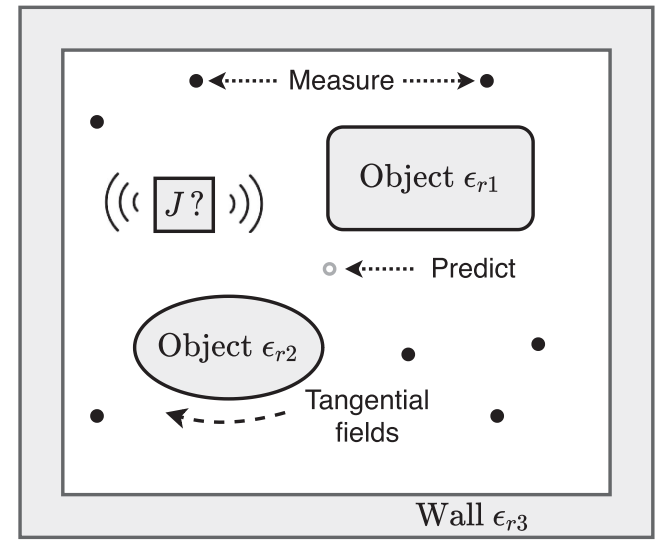

Fig. 1. Problem depiction with a source $(J)$ radiating in the presence of objects, enclosed within a wall. Field measurements are made at strategic locations as indicated by filled dark circles. The objective is to predict the field at any point outside the object(s) and within the walls. To achieve this, tangential electromagnetic fields are estimated on object and wall boundaries.

around the corners of the objects, waveguiding in the corridors, and multiple reflections [7, Fig. 3]. Another popular approach is to assume that the EM fields are sparse in the spatial Fourier domain, and to leverage this information to reconstruct the fields [8]. This assumption works well when the domain of interest is far from the scatterers and the sources. Moreover, in most of the literature on EM inverse problems, information on the scattered fields is needed to solve the problem, which implies that the incident field should also be known [9], [10].

In this paper, we present a method to reconstruct the total EM field from total field measurements at optimal sampling locations. We do not assume any prior knowledge of the incident field or the permittivities of the scatterers. However, we assume that the region between the scatterers is homogeneous and that the approximate geometry of the scatterers and the source is known. Our method is based on surface integral formulations and is therefore a significant improvement over existing ray tracing methods in the literature. In recent work [11], we presented a method for reconstructing the scattered EM fields by making measurements at random locations, but assumed knowledge of the incident field. In the current work, we generalize this method to recover the total field without any prior knowledge of the incident field. We also identify optimal sampling locations in the environment. The problem setup is graphically represented in Fig. 1. 
Our approach: The total field at a point can be expressed as the sum of the incident field and scattered field. According to the Huygens' Principle [12], the scattered field can be written in terms of the free-space Green's function and the tangential electric and magnetic fields on the scatterer surfaces. Additionally, the Extinction theorem [12] provides relations between these tangential electric and magnetic fields. Finally, Grafs' addition theorem [13, Section 9.1] is used to express the incident field in a known basis. In our method, we measure the field at various locations in the environment, using which we solve for the incident field and the tangential fields on the surfaces of the scatterers. The estimated incident field and the tangential fields are then substituted back into the Huygens' Principle to find the total field everywhere.

Central to our approach for solving this system is the idea of compressive sensing (CS), which exploits sparsity of the unknown signal in certain domains in order to recover it from fewer samples than dictated by the Shannon sampling theorem [14], [15]. Using the principles of CS, we leverage the fact that the tangential fields are sparse in the discrete Fourier transform (DFT) domain. We also employ the framework of the Subspace Optimization Method (SOM) [16], which allows us to split the solution into two orthogonal spaces; one part of the solution is recovered from the measured data, and CS is applied to recover the remaining part of the solution.

Sensor Placement: The problem of finding the optimal sampling locations is commonly referred to as 'sensor placement' in the literature. Formally, sensor placement is the selection of the 'best' $M$ sensor locations out of $P(P \gg M)$ possible locations in the environment.

The brute force way to solve the problem of sensor placement is to try out all the $\left(\begin{array}{l}P \\ M\end{array}\right)$ possibilities, and choose that combination which results in the least error (of some predefined metric). This combinatorial approach is NP-hard and is intractable even for small values of $P$ (eg. $\left(\begin{array}{l}50 \\ 25\end{array}\right)>10^{14}$ ). The problem of obtaining the optimal sensor locations at a lower computational cost has been studied extensively in the literature. Early approaches include heuristics like cross-entropy optimization [17], genetic algorithms [18], and tabu search [19]. However, these heuristics neither provide any performance bounds nor guarantee convergence to the optimal solution. [20] formulated the sensor selection problem as a non-convex optimization of the determinant of the error covariance matrix and proposed to solve a convex relaxation of it. They also provided a lower bound on the performance, which can be used to determine how suboptimal the solution is. Due to the convex relaxation, this method sometimes results in an ill-conditioned matrix, especially when the number of sensors is limited. Few greedy approaches have also been proposed based on proxies of the estimation error like condition number [21], [22], and frame potential [23]. However, these proxies may also result in an ill-conditioned matrix. Moreover, minimizing these proxies results in the optimal solution only if the rows of the observation matrix are unit norm. Jiang et al. proposed MPME (Maximal Projection on Minimum Eigenspace) [24], a greedy sensor selection algorithm that maximizes the minimum eigenvalue of the observation matrix in a computationally efficient manner. MPME outperforms condition number and frame

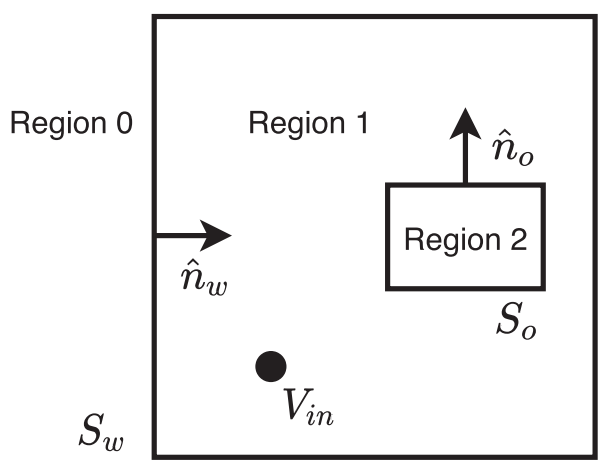

Fig. 2. Problem geometry: $S_{w}$ is the inner surface of the enclosing wall and $S_{o}$ is the outer surface of a non-magnetic scatterer. $V_{\text {in }}$ denotes the volume enclosing the source, whose distribution is unknown. $\hat{n}_{w}$ and $\hat{n}_{o}$ are the normals to the scattering surfaces of the wall and object respectively.

potential based methods (in terms of the mean squared error). Further, it does not assume that the observation vectors are unit norm. These advantages make MPME a suitable algorithm for our problem. In later sections, we show that using the optimal sampling locations returned by MPME instead of random sampling locations reduces the error by almost $50 \%$ in some cases.

Paper Organization: The rest of the paper is organized as follows. In Section II, we describe the problem statement and the mathematical formulation. In Section III, we discuss the algorithms used for sensor selection and for solving the system. Section IV contains the numerical results for sensor selection and EM field reconstruction. We conclude the paper with a discussion of the results in Section V.

\section{PROBLEM FoRMULATION}

Consider the two dimensional (2D) schematic shown in Fig. 2. For the transverse magnetic (TM) polarization, the total field at any point $\vec{r}$ in Region 1 can be expressed using Huygens' principle as [25]:

$$
\begin{aligned}
\phi(\vec{r})= & \phi_{\text {in }}(\vec{r}) \\
& -\oint_{S_{w}}\left[g\left(\vec{r}, \vec{r}^{\prime}\right) \nabla^{\prime} \phi_{w}\left(\vec{r}^{\prime}\right)-\phi_{w}\left(\vec{r}^{\prime}\right) \nabla^{\prime} g\left(\vec{r}, \vec{r}^{\prime}\right)\right] \cdot \hat{n}_{w} d l^{\prime} \\
& -\oint_{S_{o}}\left[g\left(\vec{r}, \vec{r}^{\prime}\right) \nabla^{\prime} \phi_{o}\left(\vec{r}^{\prime}\right)-\phi_{o}\left(\vec{r}^{\prime}\right) \nabla^{\prime} g\left(\vec{r}, \vec{r}^{\prime}\right)\right] \cdot \hat{n}_{o} d l^{\prime}
\end{aligned}
$$

where $\phi(\vec{r})$ is the total electric field at $\vec{r}, \phi_{\text {in }}(\vec{r})$ is the incident field, $g\left(\vec{r}, \vec{r}^{\prime}\right)=-\frac{j}{4} H_{0}^{(2)}\left(k_{0}\left|\vec{r}-\vec{r}^{\prime}\right|\right)$ is the 2D free space Green's function ( $k_{0}$ is the free space wavenumber) and $\hat{n}$ is the unit normal from the surfaces $S_{w}$ and $S_{o}$ into Region 1. $\phi_{o}, \phi_{w}$ are the tangential electric fields on the object and wall respectively, and $\nabla \phi_{o} . \hat{n}_{o}, \nabla \phi_{w} . \hat{n}_{w}$ are proportional to the tangential magnetic fields on the object and wall, respectively.

As per the Uniqueness theorem of electromagnetics [26], the field at any point in a region is uniquely specified by a specification of the source(s) and one of following over the boundaries $(\mathcal{S})$ enclosing a region: 
(a) the tangential electric field over $\mathcal{S}$, or (b) the tangential magnetic field over $\mathcal{S}$, or (c) the tangential electric field over a part of $\mathcal{S}$, and the tangential magnetic field over the remaining part.

However, in our expression of the Huygens' principle in Eq. (1), we have used both the tangential electric and magnetic fields. This redundancy is lifted when we use another relation between the tangential electric and magnetic fields, given by the Extinction theorem for Region 1 [25]:

$$
\begin{aligned}
\phi_{\text {in }}(\vec{r}) & -\oint_{S_{w}}\left[g\left(\vec{r}, \vec{r}^{\prime}\right) \nabla^{\prime} \phi_{w}\left(\vec{r}^{\prime}\right)-\phi_{w}\left(\vec{r}^{\prime}\right) \nabla^{\prime} g\left(\vec{r}, \vec{r}^{\prime}\right)\right] \cdot \hat{n}_{w} d l^{\prime} \\
& -\oint_{S_{o}}\left[g\left(\vec{r}, \vec{r}^{\prime}\right) \nabla^{\prime} \phi_{o}\left(\vec{r}^{\prime}\right)-\phi_{o}\left(\vec{r}^{\prime}\right) \nabla^{\prime} g\left(\vec{r}, \vec{r}^{\prime}\right)\right] \cdot \hat{n}_{o} d l^{\prime} \\
& =0, \quad \vec{r} \in\left\{S_{w}, S_{o}\right\} .
\end{aligned}
$$

\section{A. Expanding the Unknowns in a Basis}

The unknown quantities in Eqs. (1) and (2) are the tangential fields on the scatterers' surfaces and the incident field. Our approach is to expand the unknowns in terms of a known basis, and to estimate the coefficients of the basis functions using the field measurements.

The incident field $\phi_{\text {in }}(\vec{r})$ is expressed in terms of the current density $J(\vec{r})$ as [25],

$$
\phi_{\text {in }}(\vec{r})=-j \omega \mu_{0} \int_{V_{\text {in }}} g\left(\vec{r}, \vec{r}^{\prime}\right) J\left(\vec{r}^{\prime}\right) d V,^{\prime}
$$

where $V_{\text {in }}$ is the volume enclosing the source. Using the Grafs' addition theorem [13, Section 9.1], we simplify the above equation as,

$$
\phi_{\text {in }}(\vec{r})=\sum_{i=-\left\lfloor\left(N_{\mathrm{i}} / 2\right)\right\rfloor}^{\left\lfloor\left(N_{\mathrm{i}} / 2\right)\right\rfloor} c_{i} s_{i}(\vec{r}),
$$

$$
\begin{aligned}
& \text { where, } c_{i}=\int_{V_{\text {in }}} J_{i}\left(k_{0}\left|\vec{r}_{0}-\vec{r}^{\prime}\right|\right) e^{-j i \theta^{\prime}} J\left(\vec{r}^{\prime}\right) d V^{\prime} \\
& \text { and } s_{i}(\vec{r})=(-1)^{i+1} \frac{\omega \mu_{0}}{4} e^{j i \theta} H_{i}^{(2)}\left(k_{0}\left|\vec{r}_{0}-\vec{r}\right|\right)
\end{aligned}
$$

Here, $\vec{r}_{0}$ is the center of the region $V_{\text {in }}$ and $\theta$ is the azimuthal angle of the vector $\vec{r}_{0}-\vec{r}$. Details of this derivation are given in Appendix A.

The tangential fields are expanded in a pulse basis (see [11, Sec. 3]) as,

$$
\begin{aligned}
& \phi_{o}(r)=\sum_{i=1}^{N_{o}} a_{i}^{o} p_{i}(r), \quad \nabla \phi_{o}(r) \cdot \hat{n}_{o}=\sum_{i=1}^{N_{o}} b_{i}^{o} p_{i}(r), \\
& \phi_{w}(r)=\sum_{i=1}^{N_{w}} a_{i}^{w} q_{i}(r), \quad \nabla \phi_{w}(r) \cdot \hat{n}_{w}=\sum_{i=1}^{N_{w}} b_{i}^{w} q_{i}(r),
\end{aligned}
$$

where lowercase $r$ denotes the parametrized distance along each surface $\left(S_{o}\right.$ or $\left.S_{w}\right) . p_{i}(r), i=1,2, \ldots, N_{o}$, and $q_{i}(r)$, $i=1,2, \ldots, N_{w}$ are the sets of pulse basis functions and $a_{i}^{o}$, $b_{i}^{o}, a_{i}^{w}$ and $b_{i}^{w}$ are their corresponding unknown coefficients.
The tangential electric field on the surface of a perfect electric conductor (PEC) is zero. Therefore, if an object in the scattering domain is a PEC, we can set the corresponding coefficients $\left(\boldsymbol{a}_{i}\right.$ for the $i^{\text {th }}$ object) to zero. This reduces the number of unknowns (and therefore the number of required measurements).

\section{B. Defining the Data Equation}

Substituting Eqs. (4), (5) and (6) in Eq. (1) and making field measurements at $M$ locations, we get,

$$
\underbrace{\left[\begin{array}{lllll}
E & F & G & H & S
\end{array}\right]}_{A_{d}} \underbrace{\left[\begin{array}{c}
\boldsymbol{a}^{o} \\
\boldsymbol{b}^{o} \\
\boldsymbol{a}^{w} \\
\boldsymbol{b}^{w} \\
\boldsymbol{c}
\end{array}\right]}_{\boldsymbol{x}}=\underbrace{\left[\begin{array}{c}
\phi\left(\vec{r}_{1}\right) \\
\phi\left(\vec{r}_{2}\right) \\
\vdots \\
\phi\left(\vec{r}_{M}\right)
\end{array}\right]+\boldsymbol{\nu}}_{\boldsymbol{b}_{d}}
$$

where $\quad\{E, F\} \in \mathbb{C}^{M \times N_{o}}, \quad\{G, H\} \in \mathbb{C}^{M \times N_{w}}, \quad$ and $\quad S \in$ $\mathbb{C}^{M \times N_{\text {in }}}$ are submatrices that constitute the matrix $A_{d} \in \mathbb{C}^{M \times N}$, $x \in \mathbb{C}^{N}$ is the unknown vector formed by vertically stacking the vectors $\left\{\boldsymbol{a}^{o}, \boldsymbol{b}^{o}\right\} \in \mathbb{C}^{N_{o}},\left\{\boldsymbol{a}^{w}, \boldsymbol{b}^{w}\right\} \in \mathbb{C}^{N_{w}}$, and $\boldsymbol{c} \in \mathbb{C}^{N_{\mathrm{in}}}$; $N=2\left(N_{w}+N_{o}\right)+N_{\text {in }}$ denotes the total number of unknowns. The total field at a location $\vec{r}_{i}$, denoted by $\phi\left(\vec{r}_{i}\right)$, is corrupted by noise given in $\nu$. The elements of the submatrices $E, F, G, H$ are computed as per the relations in [11, Eq. (9)]. The elements of the submatrix $S$ are given as $s_{m, i}=s_{i}\left(\vec{r}_{m}\right)$, where $\vec{r}_{m}$ denotes the measurement location of the $m^{\text {th }}$ measurement point. We call Eq. (7) the 'Data equation' and $A_{d}$ the 'Data matrix'.

For notational convenience, we denote a row of $A_{d}$ by $\boldsymbol{a}_{d}(\vec{r})$. In terms of $\boldsymbol{a}_{d}(\vec{r})$, we can write the total field at a point $\vec{r}$ in Region 1 (in Fig. 2) as,

$$
\boldsymbol{a}_{d}(\vec{r}) \boldsymbol{x}=\phi(\vec{r})+\nu,
$$

where $\nu$ is the noise corrupting the total field measurement.

\section{Defining the State Equation}

Similarly, substituting Eqs. (4), (5) and (6) in Eq. (2), and specifying the Extinction theorem at the midpoints of the discretized edges (as defined by the pulse basis in Eqs. (5), (6)) of $S_{o}$ and $S_{w}$ (total $N_{o}+N_{w}$ in number) gives,

$$
A_{s} \boldsymbol{x}=\mathbf{0},
$$

where $\mathbf{0} \in \mathbb{R}^{N_{o}+N_{w}}$ is a vector of zeros. We call Eq. (9) the 'State equation' and $A_{s}$ the 'State matrix'.

\section{Outline of Our Method}

We propose to predict EM fields using the following approach:

1) Determine the $M$ optimal sampling locations in the region in between the scatterers (Region 1 in Fig. 2), and measure the total field at these locations.

2) Find the tangential field and incident field coefficients that satisfy the Data and State equations.

3) Substitute the recovered fields into the Huygens' principle to estimate the total field at desired locations. 


\section{METHODS}

In the previous section, we derived the data equation and the state equation and provided an outline of our approach for field prediction. In this section, we flesh out the details of the different steps in our method.

\section{A. Strategies for Choosing the Measurement Locations}

The first step in our method involves making $M$ measurements in the region in between the scatterers. A natural question arises regarding the choice of the measurement locations. The simplest answer to this question is to choose the measurement locations randomly (called random sampling or 'RS' henceforth). However, it is easy to see that random sampling is not optimal. Firstly, it could result in measurement locations being very close to each other. This would increase the number of measurements without improving the quality of the reconstruction significantly, while also degrading the numerical properties of the Data matrix. Further, it does not factor in any information about the scattering environment, or the scattering phenomenon. Therefore, by exploiting the information about the scattering environment and the physics of the problem, it is possible to come up with a better sampling scheme.

As mentioned earlier, sensor placement deals with choosing an optimal set of sensor locations from a large pool of potential locations. The latter are typically obtained by a fine spatial discretization of the scattering environment. The total field, $\boldsymbol{y}$, at the potential sampling locations $\left\{\vec{r}_{1}^{p}, \vec{r}_{2}^{p}, \ldots, \vec{r}_{P}^{p}\right\}$ can be expressed as:

$$
\underbrace{\left[\begin{array}{c}
\boldsymbol{a}_{d}\left(\vec{r}_{1}^{p}\right) \\
\boldsymbol{a}_{d}\left(\vec{r}_{2}^{p}\right) \\
\vdots \\
\boldsymbol{a}_{d}\left(\vec{r}_{P}^{p}\right)
\end{array}\right]}_{B} \boldsymbol{x}=\underbrace{\left[\begin{array}{c}
\phi\left(\vec{r}_{1}^{p}\right) \\
\phi\left(\vec{r}_{2}^{p}\right) \\
\vdots \\
\phi\left(\vec{r}_{P}^{p}\right)
\end{array}\right]+\boldsymbol{\nu}}_{\boldsymbol{y}}
$$

where $\boldsymbol{\nu} \in \mathbb{C}^{P}$ is complex additive white gaussian noise, and the matrix, $B \in \mathbb{C}^{P \times N}$, (called the 'Propagator') is constructed by row vectors of the form defined in (8). Typically, we have $P \gg N$.

Since the Propagator is constructed by finely discretizing the environment, it closely approximates the continuous space operator that maps the intermediate variables to the total field. Thus, sensor placement can be defined as selecting the best $M$ rows from the Propagator $B$ such that the estimation error is minimum. Out of the many approaches that have been proposed in the literature for sensor placement, we choose the MPME algorithm [24] which has shown the best results for sensor placement in our problem.

$M P M E v / s$ random sampling: In order to compare MPME and random sampling, we present some results for sensor placement for an indoor scenario with a single scattering object. Fig. 3 shows a comparison of the sampling points obtained from MPME and random selection. As expected, random samples are found everywhere in the region in between the scatterers whereas, the MPME samples closely circumscribe the scatterers
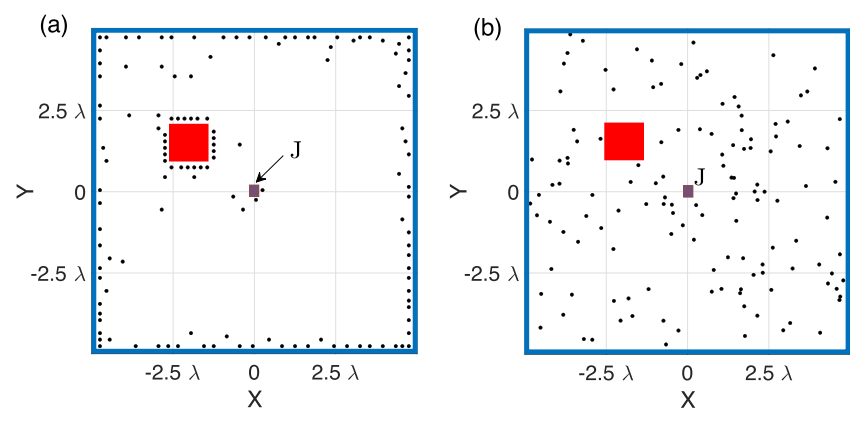

Fig. 3. Comparison of the sampling points $(M=132)$ obtained from (a) MPME and (b) random sampling schemes in an indoor scenario with a single scattering object (shown in red color). The source, $J$, is placed at the center and is shaded in brown. While random samples are scattered throughout the domain, MPME samples are spaced further apart and closer to the boundaries.

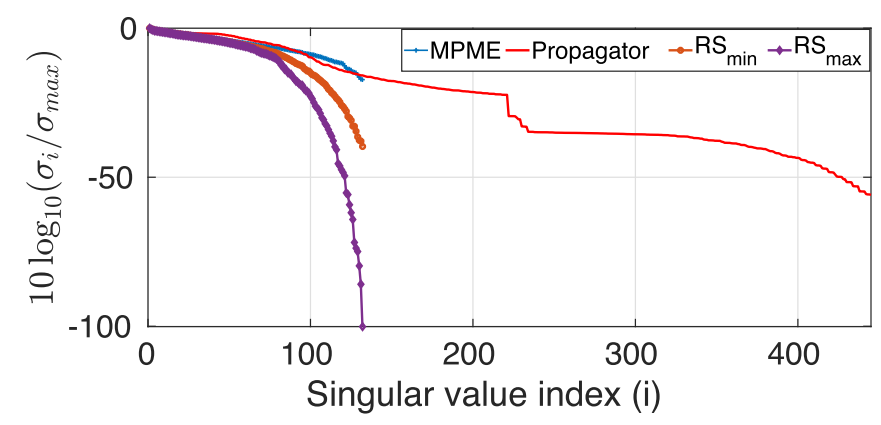

Fig. 4. Comparison of the (normalized) spectrum of the Propagator with that of the Data matrix, $A_{M}$, constructed with 132 MPME samples, and $A_{R}$ constructed with 132 random samples. For 100 different instances of random sampling, the spectrum of $A_{R}$ lies between the curves denoted by $\mathrm{RS}_{\min }$ and $\mathrm{RS}_{\max }$.

and the source. This observation is consistent with the Huygens' principle of electromagnetics [26] stated earlier in Sec. II: making measurements on a contour that closely circumscribes the scatterer surfaces amounts to (approximately) specifying the tangential electric field on the surface of the scatterer, which in turn is sufficient to compute the field outside the surface.

In order to obtain a more quantitative method for comparison, we construct Data matrices, $A_{R}$ and $A_{M}$, that correspond to the random samples and the MPME samples, respectively. As described earlier, the rows of the matrix $A_{M}$ are those rows of the Propagator, $B$, that correspond to the optimal sampling locations selected by MPME. We then compare the singular spectra of these two matrices with that of the Propagator in Fig. 4. We make two important observations from Fig. 4:

1) The spectrum of $A_{M}$ more closely approximates the spectrum of the Propagator than that of $A_{R}$, which suggests that the MPME samples approximate the Propagator better than random samples.

2) The matrix $A_{R}$ has a worse condition number than $A_{M}$. This makes field recovery using $A_{R}$ more sensitive to noise and discretization errors.

These figures are provided for illustrative purposes, and give us a good intuition as to why MPME outperforms random sampling in the detailed results that follow in Section IV. 


\section{B. Dimensionality Reduction of the Propagator}

A standard assumption in most sensor placement literature is that the number of measurements is more than the number of unknowns describing the problem, i.e. $M>N$. However, in our problem, taking more measurements than $N$ is not practical because the tangential fields are discretized on a fine scale for reasons of numerical convergence. In order to overcome this, we perform a crucial dimensionality reduction of the Propagator $B$.

To arrive at this reduction, we start by asserting that the solution $\boldsymbol{x} \in \mathbb{C}^{N}$ can be expressed in the basis given by the right singular vectors of the Propagator matrix. However, as is evident by the spectrum of the operator shown in Fig. (4), the significance of these vectors decays at higher indices of singular values. Thus, we can restrict $\boldsymbol{x}$ to a smaller sub-space of dimensionality $\zeta$ by expressing it in terms of a $\beta \in \mathbb{C}^{\zeta}$ :

$$
\boldsymbol{x}=\left[\begin{array}{llll}
\boldsymbol{w}_{1} & \boldsymbol{w}_{2} & \ldots & \boldsymbol{w}_{\zeta}
\end{array}\right] \beta=W \beta
$$

where $W \in \mathbb{C}^{N \times \zeta},\left\{\boldsymbol{w}_{i}\right\}_{i=1}^{N}$ are the right singular vectors of $B$, and the parameter $\zeta$ denotes the number of 'degrees of freedom' of the electromagnetic field [27]-[29]. The latter is defined as [30]:

$$
\zeta=\min \left(m: \frac{\sum_{i=1}^{m}\left|\sigma_{B, i}\right|^{2}}{\sum_{i=1}^{N}\left|\sigma_{B, i}\right|^{2}} \geq f\right),
$$

where $\sigma_{B, i}$ are the singular values of $B$ arranged in descending order, and the value of $0<f<1$ is chosen heuristically. We find that the set of locations returned by MPME is not very sensitive to small variations in the value of $f$. Thus, we can speak of an 'effective' Propagator given by $B^{\prime}=B W$, and by restricting $\zeta<M$, we can perform the sensor placement algorithm, MPME, on $B^{\prime}$ instead. Note that we only use the concept of dimensionality reduction for the purpose of finding out the optimal sensing locations.

\section{Solving for the Tangential Field and Incident Field Coefficients}

We now discuss the algorithm used to solve for the vector $\boldsymbol{x}$. In our earlier work [11], we proposed CS-SOM to solve for the tangential fields using scattered field measurements. The method was based on the Subspace optimization method (SOM) [16], and exploited the principles of Compressive sensing (CS) to achieve low reconstruction errors. In the current paper, we adapt CS-SOM to the case where total field measurements are considered and call this algorithm 'Total field - Compressive sensing based subspace optimization method' (TCS-SOM). In the rest of this section, we elaborate the different stages of TCS-SOM. In the framework of SOM, we split the solution space into two orthogonal spaces, viz. the major space and the minor space. ${ }^{1}$ While the major space is spanned by the top $L_{0}$ right singular vectors of the Data matrix, $A_{d}$, the minor space is spanned by the remaining $N-L_{0}$ right singular vectors, where $L_{0}<M$. We then recover the component of the solution in each of these two spaces separately.

\footnotetext{
${ }^{1}$ In our earlier work, we referred to these vector spaces as the signal and noise spaces, respectively.
}

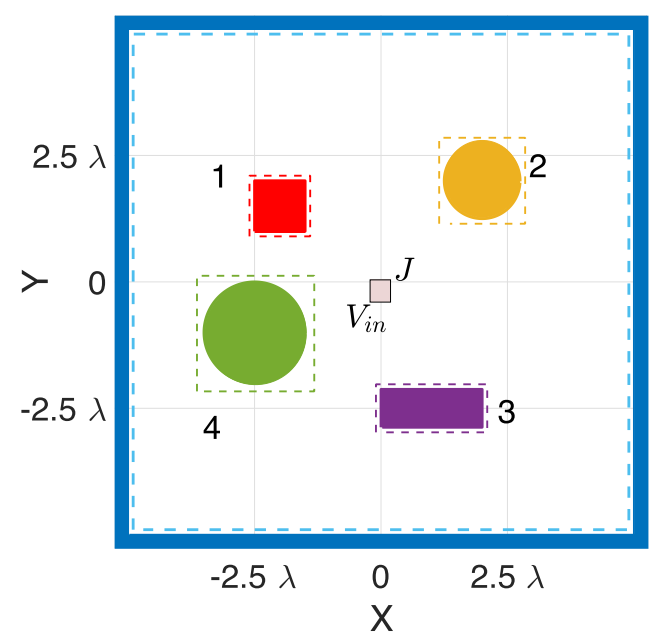

Fig. 5. The schematic of a $10 \lambda \times 10 \lambda$ simulation domain, where $\lambda$ is the wavelength of the incident wave in free space. The domain includes a wall, 4 objects and a source. Object 1 is a square of side $\lambda$, object 2 is a circle with radius $0.75 \lambda$, object 3 is a rectangle with sides $1 \lambda \times 2.5 \lambda$ and object 4 is a circle with radius $1 \lambda$. The relative permittivities of respective objects are $\epsilon_{r 1}=$ $3.7-2.1 j, \epsilon_{r 2}=1.7-1.1 j, \epsilon_{r 3}=2.7-3.7 j, \epsilon_{r 4}=1.2-1.1 j$ and the relative permittivity of the wall is $\epsilon_{r w}=3.7-2.1 j$. The objects $1,2,3$ and 4 are centered at $(-2.5 \lambda, 1.5 \lambda),(2 \lambda, 2 \lambda),(1 \lambda,-2.5 \lambda)$ and $(-2.5 \lambda,-1 \lambda)$. The source is placed within a $\lambda / 4 \times \lambda / 4$ square that is centered at origin. The exact shape of the objects are indicated by the filled regions and the dotted contours are the approximate object contours used in the problem solution.

Major space component: Consider the singular value decomposition (SVD) of the Data matrix, $A_{d}=\sum_{i} \boldsymbol{u}_{i} \sigma_{i} \boldsymbol{v}_{i}^{H}$ where $\left\{\boldsymbol{u}_{i}\right\}_{i=1}^{M},\left\{\boldsymbol{v}_{i}\right\}_{i=1}^{N}$ are the left and right singular vectors of $A_{d}$ respectively, and $\left\{\sigma_{i}\right\}_{i=1}^{M}$ are the singular values of $A_{d}$ arranged in descending order. The major space component, $\boldsymbol{x}_{1}$ is recovered using the truncated SVD solution given by,

$$
\boldsymbol{x}_{1}=\sum_{i=1}^{L_{0}}\left(\frac{\boldsymbol{u}_{i}^{H} \boldsymbol{b}_{d}}{\sigma_{i}}\right) \boldsymbol{v}_{i}
$$

The value of $L_{0}$ is chosen using the Morozov Discrepancy Principle [31] as,

$$
L_{0}=\min \left(l:\left\|A_{d}\left[\sum_{i=1}^{l}\left(\frac{\boldsymbol{u}_{i}^{H} \boldsymbol{b}_{d}}{\sigma_{i}}\right) \boldsymbol{v}_{i}\right]-\boldsymbol{b}_{d}\right\|_{2}<\epsilon\right)
$$

where $\epsilon$ is the square root of the variance of the noise, i.e. $\epsilon=$ $\left\|\boldsymbol{b}_{d}\right\| / 10^{(\mathrm{SNR} / 20)}$, where SNR is the signal to noise ratio in $\mathrm{dB}$. We have observed that the estimation error is not very sensitive to the choice of $L_{0}$, which is in agreement with other studies in literature [16], [32].

Minor space component: The minor space is spanned by the bottom-most $N-L_{0}$ right singular vectors. This component cannot be recovered by the truncated SVD method. However, as we have observed in our previous work [11, Fig. 2], the tangential fields on the surfaces of the scatterers are sparse in the DFT domain. This observation allows us to exploit the principles of Compressive Sensing (CS) [14] to recover the minor space component. In fact, [33, Fig. 6.14] shows that enforcing sparsity is a general principle in all problems involving the radiation operator. This is because the right singular vectors of the radiation operator corresponding to the large singular values 

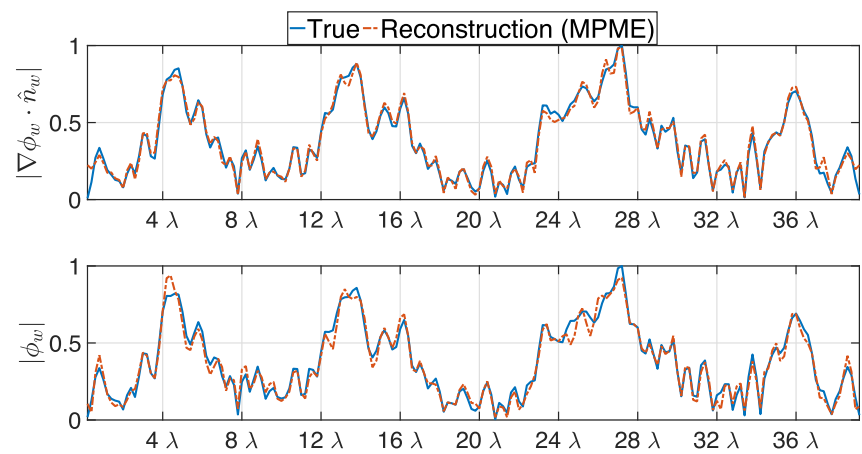

Fig. 6. The normalized magnitudes of the estimated tangential fields for MPME samples (at $0.3 \mathrm{SR}$ ) on the inexact wall, along with the true tangential fields. The measurements have an SNR of $25 \mathrm{~dB}$. The tangential fields are estimated with $\Delta_{T} 17 \%$ error.

resemble the low frequency Fourier bases, whereas singular vectors corresponding to the small singular values resemble the high frequency bases.

The optimization problem to be solved: We denote the minor space component by $\boldsymbol{x}_{2}$. Keeping $\boldsymbol{x}_{1}$ constant, we solve the following convex optimization problem that is constrained using both the Data and State equations to recover $\boldsymbol{x}_{2}$ :

$$
\begin{array}{cc}
\underset{\boldsymbol{x}_{2}}{\operatorname{minimize}} & \left\|\mathcal{M} I_{1}\left(\boldsymbol{x}_{1}+\boldsymbol{x}_{2}\right)\right\|_{1}+t\left\|I_{2}\left(\boldsymbol{x}_{1}+\boldsymbol{x}_{2}\right)\right\|_{2} \\
\text { subject to } & \left\|A_{d}\left(\boldsymbol{x}_{1}+\boldsymbol{x}_{2}\right)-\boldsymbol{b}_{d}\right\|_{2} \leq \epsilon, \\
& \left\|A_{s}\left(\boldsymbol{x}_{1}+\boldsymbol{x}_{2}\right)\right\|_{2} \leq \eta
\end{array}
$$

where $\mathcal{M}=\mathcal{F}$ or $\mathcal{D}$ is the discrete fourier transform (DFT) or the discrete cosine transform (DCT) basis respectively, chosen as candidates for sparse representations, $I_{1} \in \mathbb{R}^{2\left(N_{o}+N_{w}\right) \times N}$ and $I_{2} \in \mathbb{R}^{N_{\mathrm{i}} \times N}$ are blockwise identity matrices which when multiplied with a $N$ dimensional vector return the first $2\left(N_{o}+N_{w}\right)$ and last $N_{\mathrm{i}}$ elements of that vector respectively, and $t$ is a regularization parameter; $\eta$ is an estimate of the discretization error in the state equation. We solve the problem (Eq. (15)) using the CVX package [34], [35].

\section{NUMERICAL RESULTS}

In this Section, we present the results of various numerical simulations to evaluate the performance of the proposed method. All the simulations are programmed in MATLAB R2019b on a 2.4 GHz Quad-Core Intel Core i5 processor, using 8 GB RAM.

\section{A. Simulation Setup}

The simulation domain mimics an indoor scenario with a wall enclosing objects of various sizes and permittivities as seen in Fig. 5. The exact shape and permittivites of the objects are only required to generate the synthetic measurements using a forward solver. The dotted contours, which approximate the spatial support of the objects, are the only object-specific information provided to the field reconstruction algorithm. The approximate contours are no closer than a distance of $0.1 \lambda$ from the exact contours, and measurement locations are chosen at a distance of at least $0.1 \lambda$ from the approximate contours.
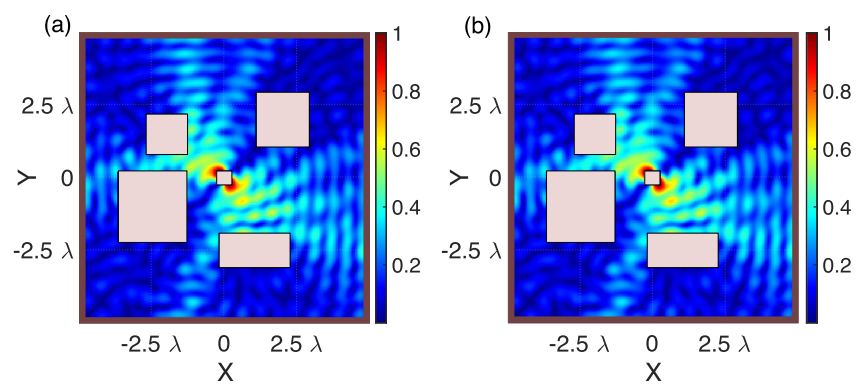

Fig. 7. The normalized magnitudes of (a) true and (b) reconstructed $2 \mathrm{D}$ total fields over a $10 \lambda \times 10 \lambda$ obtained from an SR of 0.3 (194 measurements). The sampling locations are obtained by MPME and the measurements have an SNR of $25 \mathrm{~dB}$. The colorbar shows the field magnitude in $\mathrm{V} / \mathrm{m}$.

\section{B. Source Function}

The source current, $J$, is placed within a region $V_{\text {in }}$ (see Fig. 5) at the center of the domain. We consider the following $z$-directed source current for all our simulations:

$$
J(x, y)= \begin{cases}I_{0}\left(x^{2}+y^{2}-x+y\right) & (x, y) \in V_{\text {in }} \\ 0 & \text { else }\end{cases}
$$

where $I_{0}$ is a normalization constant such that the magnitude of the incident field at a radius of $10 \lambda$ is approximately unity. A $6 \times 6$ Gauss Legendre quadrature rule in $2 \mathrm{D}$ is used to calculate the true incident field for generating synthetic measurements (by substituting Eq. (16) into Eq. (3)). We stress that the reconstruction algorithm works for any general source distribution; it is only for illustrative purposes that we have considered the above asymmetric current distribution.

\section{Generate Synthetic Measurements: Forward Solver}

The total field measurements are synthetically generated using a Boundary Integral (BI) solver [25] with pulse basis functions discretized at $\lambda / 40$, and delta testing functions. The frequency of operation is $1.5 \mathrm{GHz}$. Fig. 7 shows the magnitude of the true total EM field for the above simulation setup. The permittivity and the exact geometry of the objects are used only in the forward solver. We validate the BI solver with the Mie series solution for scattering from a single infinite cylinder of a radius $\lambda$. We also verify that there are no internal resonances around the operating frequency.

\section{Inverse Solver}

The dotted contours that represent the approximate object geometries are discretized at a value of $\lambda / 5$. This value is empirically chosen and provides an optimal trade-off between accuracy and computational cost. The number of unknown variables obtained by discretizing the inexact surfaces of wall and four objects (in that order) are: $2 \times(196+24+32+30+42)=648$. The source is enclosed in a square of side $\lambda / 4$, which gives the number of coefficients (see Appendix A, Eq. (23)) as $N_{i}=5$. Therefore, the total number of variables are $N=653$. To find the degrees of freedom, we (heuristically) set $f=0.999$ i.e. the first $\zeta$ singular values that account for $99.9 \%$ of Eq. (12). Using 
this value of $\zeta$, we run the MPME algorithm to get the optimal sampling locations.

The Data matrices $A_{M}$ and $A_{R}$ are constructed from the sampling locations obtained from MPME and random sampling (RS) schemes. At the sampling locations, we measure the total field, which is corrupted by additive white gaussian noise (AWGN). We consider two different noise levels corresponding to $25 \mathrm{~dB}$ and $10 \mathrm{~dB}$ signal to noise ratio (SNR) and report the estimation errors for different number of measurements. We also report the sampling rate (SR), which is defined as the ratio of the number of measurements to the total number of unknowns. The major part of the solution (see Eq. (13)) is estimated using the Morozov discrepancy principle. To get the minor part, we solve Eq. (15) with the DCT as the sparsifying transform. The regularization parameter, $t$ in Eq. (15) is set to 1 . However, we note that the algorithm is not very sensitive to the choice of $t$. In our simulations, the value of the discretization error in the State equation (obtained using the forward solver) is $\left\|A_{s} \boldsymbol{x}\right\| \approx 0.03$. Therefore, we set $\eta=0.1$. We note that in general, setting $\eta=0.1$ is a good rule of thumb, and gives good reconstructions in most of the extensive numerical simulations that we have studied.

The computational complexity of the MPME algorithm is $\mathcal{O}\left(P M N^{2}\right)$ [24], and it takes $144 \mathrm{~s}$ to identify the optimal sampling locations. We average our results for both MPME and random sampling over 100 Monte carlo iterations. The run time for a single iteration of the inverse solver is less than 2 minutes. This includes the major space estimation $\left(\mathcal{O}\left(M^{2}+M N\right)\right)$, the minor space estimation $\left(\mathcal{O}\left(N^{2} M^{2.5}+M^{3.5}\right)\right)$, and field prediction $(\mathcal{O}(Q N)-Q$ is number of points where the field is predicted). For predicting the field, we precompute a prediction matrix. The construction of this matrix takes around 30 minutes. However, this is a one time cost. Overall, the total time taken for 100 Monte Carlo iterations for MPME sampling is around 4 hours, and for random sampling is around 4.5 hours.

\section{E. Error Definition}

To test the accuracy of our algorithm, we compare the reconstructed and true fields and define three error metrics as: tangential field error $\Delta_{T}, 2 \mathrm{D}$ grid error $\Delta_{G}$, and the incident field error $\Delta_{I}$.

The tangential field error $\left(\Delta_{T}\right)$ is defined as:

$$
\Delta_{T}=\frac{\|\hat{\boldsymbol{x}}-\boldsymbol{x}\|_{2}}{\|\boldsymbol{x}\|_{2}},
$$

where $\hat{\boldsymbol{x}}$ and $\boldsymbol{x}$ are the estimated and true tangential fields respectively. Once the incident field and the tangential coefficients are estimated we substitute them in Eq. (1) to get the field over the $10 \lambda \times 10 \lambda$ region (discretized on a grid with pitch equal to $\lambda / 20)$. The error in field prediction over this grid is defined as:

$$
\Delta_{G}=\frac{\|\hat{\phi}-\phi\|_{2}}{\|\phi\|_{2}},
$$

where $\hat{\phi}$ and $\phi$ are the estimated and true total fields over the 2D grid of points respectively. Finally, the error in incident field
TABLE I

\begin{tabular}{|c|c|c|c|c|c|c|c|}
\hline \multirow{3}{*}{ Scheme } & & \multicolumn{6}{|c|}{ Measurements/ Sampling rate } \\
\hline & & \multicolumn{3}{|c|}{$194 / 0.3 x$} & \multicolumn{3}{|c|}{$356 / 0.55 x$} \\
\hline & & $\Delta_{T}$ & $\Delta_{G}$ & $\Delta_{I}$ & $\Delta_{T}$ & $\Delta_{G}$ & $\Delta_{I}$ \\
\hline \multicolumn{8}{|c|}{ SNR : $25 \mathrm{~dB}$} \\
\hline \multirow{2}{*}{ MPME } & Mean & 19 & 8 & 5 & 17 & 5 & 4 \\
\hline & SD & 1 & 1 & 1 & 1 & 1 & 1 \\
\hline \multirow{2}{*}{$\mathrm{RS}$} & Mean & 30 & 15 & 8 & 20 & 6 & 4 \\
\hline & $\mathrm{SD}$ & 4 & 2 & 4 & 3 & 2 & 4 \\
\hline \multicolumn{8}{|c|}{ SNR : $10 \mathrm{~dB}$} \\
\hline \multirow{2}{*}{ MPME } & Mean & 38 & 30 & 23 & 38 & 26 & 16 \\
\hline & SD & 2 & 2 & 5 & 2 & 2 & 3 \\
\hline \multirow{2}{*}{$\mathrm{RS}$} & Mean & 55 & 36 & 25 & 44 & 32 & 20 \\
\hline & SD & 10 & 6 & 8 & 8 & 6 & 7 \\
\hline
\end{tabular}

COMPARISON OF ERROR FROM MPME AND RS

Percentage error in the estimated tangential field $\left(\Delta_{T}\right)$, predicted field $\left(\Delta_{G}\right)$ and incident field $\left(\Delta_{I}\right)$ over a $10 \lambda \times 10 \lambda$ grid, for four objects with different sampling rates and SNR values. The error is calculated for 100 monte carlo iterations. SD is the standard deviation.

estimation is defined as:

$$
\Delta_{I}=\frac{\left\|\hat{\phi}_{i}-\phi_{i}\right\|_{2}}{\left\|\phi_{i}\right\|_{2}},
$$

where $\hat{\phi}_{i}$ and $\phi_{i}$ are the estimated and true incident fields over the $2 \mathrm{D}$ grid of points respectively.

We also define the relative error at a location $\vec{r}$ as:

$$
\frac{|\hat{\phi}(\vec{r})-\phi(\vec{r})|}{|\phi(\vec{r})|}
$$

where $\hat{\phi}(\vec{r})$ and $\phi(\vec{r})$ are the estimated and true fields at $\vec{r}$, respectively. For the purpose of error calculation, we do not consider locations internal to the scatterers, and locations that are at a distance less than $\lambda / 10$ from the scatterer surface.

\section{F. Field Prediction Results : MPME vs RS}

Table I shows the reconstruction errors for both MPME and RS schemes for different SR and noise levels. Measurements done using locations obtained from MPME performs better than RS.

To study how the results generalize, we study the error performance for a different number of objects. Table II shows the reconstruction errors for 2 and 4 objects for a fixed SR of 0.3 . The reconstruction errors reported are averaged over 100 Monte Carlo iterations. Although not reported here, we have verified that the errors for 1 and 3 objects follow a similar trend as has been shown for 4 objects with respect to SNR and sampling ratio.

The estimated tangential fields on the wall for MPME sampling locations, 0.3 SR (194 noisy measurements with SNR of $25 \mathrm{~dB}$ ), are plotted along with the true tangential fields in Fig. 6.

The tangential field error $\left(\Delta_{T}\right)$ in this case is $17 \%$. Figs. 7 and 8 show the magnitude and phase, respectively, of the true and reconstructed field for $\mathrm{SR}=0.3$ over the entire $2 \mathrm{D}$ domain 
TABLE II

ERROR FOR VARYING NUMBER OF SCATTERERS

\begin{tabular}{|l|l|l|l|l|l|l|l|}
\hline \multirow{2}{*}{ SNR } & \multirow{6}{*}{} & \multicolumn{6}{|c|}{ Number of Objects } \\
\cline { 3 - 8 } & & \multicolumn{3}{|c|}{2} & \multicolumn{3}{c|}{4} \\
\cline { 3 - 8 } & $\Delta_{T}$ & $\Delta_{G}$ & $\Delta_{I}$ & $\Delta_{T}$ & $\Delta_{G}$ & $\Delta_{I}$ \\
\hline \multirow{2}{*}{$25 \mathrm{~dB}$} & Mean & 17 & 10 & 6 & 19 & 8 & 5 \\
\cline { 2 - 8 } & SD & 2 & 1 & 2 & 1 & 1 & 1 \\
\hline \multirow{2}{*}{$10 \mathrm{~dB}$} & Mean & 35 & 32 & 24 & 38 & 30 & 23 \\
\cline { 2 - 8 } & SD & 3 & 3 & 5 & 2 & 2 & 5 \\
\hline
\end{tabular}

Percentage error in the estimated tangential field $\left(\Delta_{T}\right)$, predicted field $\left(\Delta_{G}\right)$ and incident field $\left(\Delta_{I}\right)$ over a $10 \lambda \times 10 \lambda$ grid, for different number of objects at 0.3 sampling rate. The error is calculated for 100 monte carlo iterations. SD is the standard deviation. The number of objects are considered in serial wise as shown in Fig. 5 .
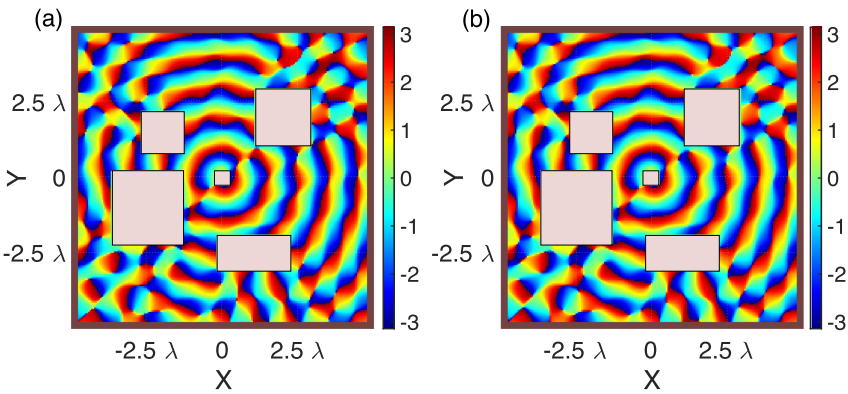

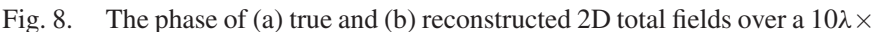
$10 \lambda$ obtained from an SR of 0.3 (194 measurements). The sampling locations are obtained by MPME and the measurements have an SNR of $25 \mathrm{~dB}$. The colorbar shows the phase in radians.
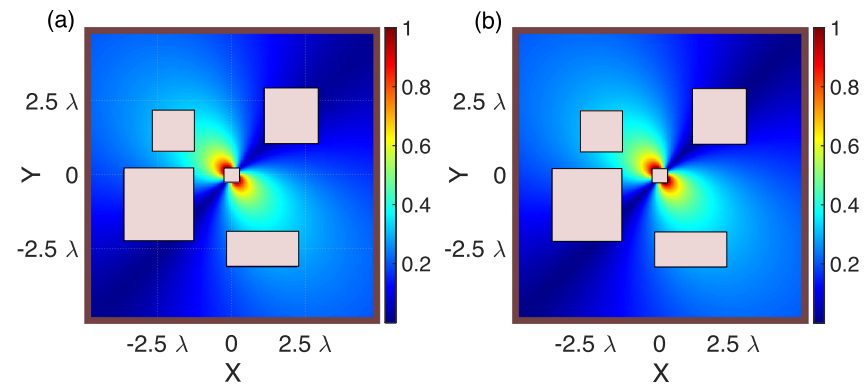

Fig. 9. The normalized magnitude of (a) true and (b) reconstructed 2D incident fields over a $10 \lambda \times 10 \lambda$ obtained from an SR of 0.3 (194 measurements). The sampling locations are obtained by MPME and the measurements have an SNR of $25 \mathrm{~dB}$. The colorbar shows the field magnitude in $\mathrm{V} / \mathrm{m}$.

outside the scattering objects. The reconstruction error on the grid for this case is $\Delta_{G}=8 \%$.

For the same measurement setup, the incident field is estimated with a very good accuracy of 5\%. Fig. 9 shows a comparison between the true and reconstructed incident fields for the two sampling schemes.

Finally, in order to study the locations in the domain that contribute the most error, we report a plot of relative total-field error in Fig. 10.

Error analysis: From the results in Table I, it can be seen that MPME outperforms random sampling and has a better field reconstruction accuracy. It turns out that the major space of $A_{M}$ has a much higher dimension than the major space of $A_{R}$ (This
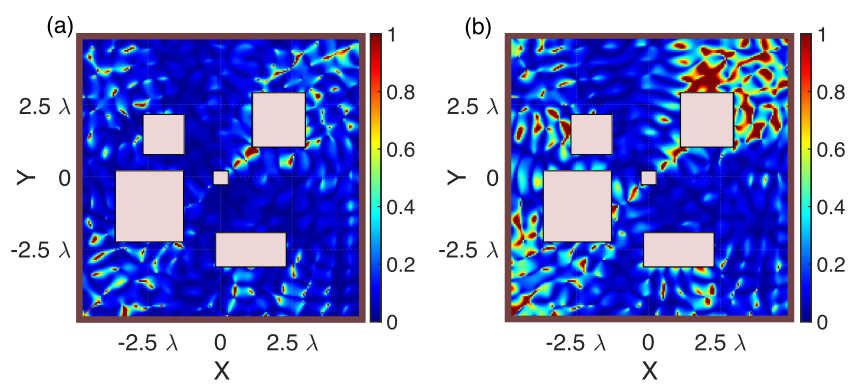

Fig. 10. The relative error in prediction of total fields over a $10 \lambda \times 10 \lambda$ obtained from an SR of 0.3 (194 measurements) (a) MPME and (b) RS measurement. The measurements have an SNR of $25 \mathrm{~dB}$. The colorbar shows the relative error

can be seen in Fig. 4, where the magnitude of the normalized singular values of $A_{M}$ is greater than that of $A_{R}$ ). It is generally observed that the error in reconstructing the major space components is lower than the error encountered in the minor space components, provided sufficient data (measurements) is available. We find that the major space recovery is of comparable accuracy between the MPME and RS approaches. However, the minor space recovery is found to be more accurate in the case of MPME as compared to the RS approach. Due to this, the error in reconstruction of the tangential field and the incident field coefficients is much lower for the MPME approach.

\section{DISCUSSION}

Summary: In this paper, we have proposed a method for recovering the total field in an arbitrary scattering environment by making a few measurements of the electromagnetic field. No assumptions about the permittivities of the scatterers or the source distribution are made, though the region in between the scatterers is assumed to be homogeneous and the approximate geometries of the scatterers and the source are also assumed to be known. Further, we have used the MPME algorithm to identify the optimal locations for making field measurements. Using this method we have recovered the total field with an average error of $8 \%$ for a sampling ratio (SR) of 0.3 and an SNR of $25 \mathrm{~dB}$. The tangential fields are recovered with an error of $17 \%$ and the incident field is recovered with an error of 5\%. We have also shown that by making field measurements at the MPME locations instead of random locations, the error in total field reconstruction can be reduced by almost half at lower SRs. At higher SRs, this improvement becomes less significant.

On why MPME does better than random sampling: In Section III-A, we provided evidence to suggest that the MPME samples will perform better than random samples (see Fig. 4 and Table I). Now, we provide a physical reason to support this. The tangential fields on the scattering surfaces also produce nearfields that are confined in the proximity of these surfaces. These fields diminish as we move away from the scatterers [25]. As seen in Fig. 3 b, the random samples are scattered throughout the interior of the domain and the samples which are far away from the scattering surface fail to capture this near field information. The optimal sensor placement algorithm, MPME, picks out the best locations that are close to the scattering surfaces. The near 
field information obtained from the optimal samples helps to achieve up to twice the accuracy compared with RS for the same number of measurements.

Limitations of our method: Our proposed method suffers from the following limitations:

- One of the objectives of MPME is to choose the optimal locations such that the mean square error in the unknown vector, $\boldsymbol{x}$ lies below a certain bound. While this error $\left(\Delta_{T}\right)$ is of importance, we are also interested in the error in the reconstructed $2 \mathrm{D}$ field $\left(\Delta_{G}\right)$. No bounds can be provided for this quantity and therefore we cannot guarantee optimality in terms of $\Delta_{G}$.

- It was mentioned in Section III-A that a dimensionality reduction of the Propagator is done, using the concept of the number of degrees of freedom (DoF). In general, DoF is an SNR dependent quantity [30, Section 9]. However, our current definition (see Eq. (12)) is independent of the SNR. While this works well for higher SNRs, at lower SNRs, we notice a degradation in performance.

- The number of unknowns in our problem depends on the number of scatterers, i.e. if there are more scatterers, we need more basis functions to expand the tangential fields and therefore more unknowns. If there are more number of unknowns, we need more measurements to recover them. Therefore, as the number of scatterers increase, the number of measurements needed to recover the fields will also increase.

Extensions of this work: In many situations, it is expensive (and sometimes impossible) to take measurements with phase [36]-[39]. The problem of phase retrieval is non convex, and therefore more difficult to solve than the current problem at hand. Many successful approaches have been proposed in literature for phase retrieval [40]-[45]. One of the immediate extensions of our work concerns the recovery of EM fields from phaseless measurements.

It was mentioned in Section III-A that the MPME samples closely circumscribe the scatterers and the source, and that this is a consequence of the Huygens' principle. In future versions of our work, we plan to exploit this observation in order to make the process of sensor placement computationally efficient.

We also plan to extend the current work to vectorial and three dimensional scenarios.

\section{APPENDIX A}

\section{DERIVATION OF EQ. 4}

The incident field $\phi_{\text {in }}(\vec{r})$ in Eq. (1) can be written in terms of the source $J(\vec{r})$ with support $V_{\text {in }}$ as:

$$
\phi_{\text {in }}(\vec{r})=-j \omega \mu_{0} \int_{V_{\text {in }}} g\left(\vec{r}, \vec{r}^{\prime}\right) J\left(\vec{r}^{\prime}\right) d V^{\prime}
$$

Graf's addition theorem [13, Section 9.1] states that,

$$
H_{0}^{(2)}\left(\eta\left|\vec{r}_{j}\right|\right)=\sum_{i=-\infty}^{\infty} H_{i}^{(2)}\left(\eta\left|\vec{R}_{j l}\right|\right) J_{i}\left(\eta\left|\vec{r}_{l}\right|\right) e^{j i\left(\pi-\theta_{l}+\theta_{j l}\right)}
$$

where $\vec{r}_{j}$ and $\vec{r}_{l}$ are two position vectors, $\vec{R}_{j l}=\vec{r}_{l}-\vec{r}_{j}, \theta_{l}$ and $\theta_{j l}$ are the azimuthal angles of $\vec{r}_{l}$ and $\vec{R}_{j l}$ respectively, $H_{i}^{(2)}$ is the $i^{\text {th }}$ order Hankel function of the second kind and $J_{i}$ is the $i^{\text {th }}$ order bessel function of the first kind. Note that Eq. (22) is valid only for $\left|\vec{r}_{l}\right|<\left|\vec{R}_{j l}\right|$.

Using this theorem, the Green's function can be expanded as,

$$
\begin{aligned}
& g\left(\vec{r}, \vec{r}^{\prime}\right)=-\frac{j}{4} H_{0}^{(2)}\left(k_{0}\left|\vec{r}-\vec{r}^{\prime}\right|\right) \\
& =-\frac{j}{4} \sum_{i=-\infty}^{\infty}\left(H_{i}^{(2)}\left(k_{0}\left|\vec{r}_{0}-\vec{r}\right|\right) J_{i}\left(k_{0}\left|\vec{r}_{0}-\vec{r}^{\prime}\right|\right) e^{j i\left(\pi-\theta^{\prime}+\theta\right)}\right)
\end{aligned}
$$

where $\vec{r}_{0}$ is the center of the region $V_{\text {in }}, \vec{r}_{j}=\vec{r}-\vec{r}^{\prime}$, and $\theta^{\prime}$ and $\theta$ are the azimuthal angles of $\vec{r}_{l}=\vec{r}_{0}-\vec{r}^{\prime}$ and $\vec{R}_{j l}=\vec{r}_{l}-$ $\vec{r}_{j}=\vec{r}_{0}-\vec{r}$ respectively. The above expression is substituted in Eq. (21) to get

$$
\begin{array}{r}
\phi_{\text {in }}(\vec{r})=-\frac{\omega \mu_{0}}{4} \int_{V_{\text {in }}} \sum_{i=-\infty}^{\infty}\left[\left(H_{i}^{(2)}\left(k_{0}\left|\vec{r}_{0}-\vec{r}\right|\right) e^{j i(\pi+\theta)}\right)\right. \\
\left.J_{i}\left(k_{0}\left|\vec{r}_{0}-\vec{r}^{\prime}\right|\right) e^{-j i \theta^{\prime}}\right] J\left(\vec{r}^{\prime}\right) d V^{\prime}
\end{array}
$$

Since $H_{i}^{(2)}\left(k_{0}\left|\vec{r}_{0}-\vec{r}\right|\right) e^{j i(\pi+\theta)}$ is independent of the integration variable, we can take it out of the integral and simplify the above expression as,

$$
\begin{aligned}
\phi_{\text {in }}(\vec{r})= & \sum_{i=-\infty}^{\infty}\left((-1)^{i+1} \frac{\omega \mu_{0}}{4} e^{j i \theta} H_{i}^{(2)}\left(k_{0}\left|\vec{r}_{0}-\vec{r}\right|\right)\right. \\
& \left.\int_{V_{\text {in }}} J_{i}\left(k_{0}\left|\vec{r}_{0}-\vec{r}^{\prime}\right|\right) e^{-j i \theta^{\prime}} J\left(\vec{r}^{\prime}\right) d V^{\prime}\right)
\end{aligned}
$$

Setting $c_{i}=\int_{V_{\text {in }}} J_{i}\left(k_{0}\left|\overrightarrow{r_{0}}-\vec{r}^{\prime}\right|\right) e^{-j i \theta^{\prime}} J\left(\vec{r}^{\prime}\right) d V^{\prime}$ and $s_{i}(\vec{r})=$ $(-1)^{i+1} \frac{\omega \mu_{0}}{4} e^{j i \theta} H_{i}^{(2)}\left(k_{0}\left|\overrightarrow{r_{0}}-\vec{r}\right|\right)$,

$$
\phi_{\text {in }}(\vec{r})=\sum_{i=-\infty}^{\infty} s_{i}(\vec{r}) c_{i}
$$

When the volume $V_{\text {in }}$ lies inside a circle of radius $a$, the infinite summation can be truncated to $N_{\mathrm{i}}=\left\lceil 2 k_{0} a+1\right\rceil$ terms [46]. Using this result, we finally arrive at Eq. (4):

$$
\phi_{\text {in }}(\vec{r})=\sum_{i=-\left\lfloor\left(N_{\mathrm{i}} / 2\right)\right\rfloor}^{\left\lfloor\left(N_{\mathrm{i}} / 2\right)\right\rfloor} c_{i} s_{i}(\vec{r})
$$

\section{REFERENCES}

[1] T. B. Hansen, R. A. Marr, U. H. Lammers, T. J. Tanigawa, and R. V. McGahan, "Bistatic RCS calculations from cylindrical near-field measurements-Part I: Theory," IEEE Trans. Antennas Propag., vol. 54, no. 12 , pp. 3846-3856, Dec. 2006.

[2] L. Pei, R. Chen, J. Liu, H. Kuusniemi, T. Tenhunen, and Y. Chen, "Using inquiry-based bluetooth RSSI probability distributions for indoor positioning," J. Global Positioning Syst., vol. 9, no. 2, pp. 122-130, 2010.

[3] Z.-H. Wu, Y. Han, Y. Chen, and K. R. Liu, "A time-reversal paradigm for indoor positioning system," IEEE Trans. Veh. Technol., vol. 64, no. 4, pp. 1331-1339, Apr. 2015.

[4] A. Bose and C. H. Foh, "A practical path loss model for indoor wifi positioning enhancement," in Proc. 6th Int. Conf. Inf. Commun. Signal Process., 2007, pp. 1-5.

[5] A. Toscano, F. Bilotti, and L. Vegni, "Fast ray-tracing technique for electromagnetic field prediction in mobile communications," IEEE Trans. Magn., vol. 39, no. 3, pp. 1238-1241, May 2003. 
[6] V. Degli-Esposti, D. Guiducci, A. de'Marsi, P. Azzi, and F. Fuschini, "An advanced field prediction model including diffuse scattering," IEEE Trans. Antennas Propag., vol. 52, no. 7, pp. 1717-1728, Jul. 2004.

[7] K. A. Remley, H. R. Anderson, and A. Weisshar, "Improving the accuracy of ray-tracing techniques for indoor propagation modeling," IEEE Trans. Veh. Technol., vol. 49, no. 6, pp. 2350-2358, Nov. 2000.

[8] A. C. Austin and M. J. Neve, "Efficient field reconstruction using compressive sensing," IEEE Trans. Antennas Propag., vol. 66, no. 3, pp. 1624-1627, Mar. 2018.

[9] K. Belkebir and M. Saillard, "Testing inversion algorithms against experimental data," Inverse Problems, vol. 17, no. 6, 2001, Art. no. 1565

[10] K. Belkebir and M. Saillard, "Testing inversion algorithms against experimental data: Inhomogeneous targets," Inverse Problems, vol. 21, no. 6, pp. S1-S3, 2005.

[11] C. Bhat, K. Sastry, and U. K. Khankhoje, "Compressive sensing approaches for the prediction of scattered electromagnetic fields," J. Opt. Soc. Amer. A, vol. 37, no. 7, pp. 1166-1174, 2020.

[12] C. A. Balanis, Advanced Engineering Electromagnetics. Hoboken, NJ, USA: Wiley, 1999.

[13] M. Abramowitz and I. A. Stegun, Handbook of Mathematical Functions With Formulas, Graphs, and Mathematical Tables, vol. 55. Washington DC, USA: Courier Corporation, U.S. Government Printing Office, 1965.

[14] E. J. Candès and M. B. Wakin, "An introduction to compressive sampling [a sensing/sampling paradigm that goes against the common knowledge in data acquisition]," IEEE Signal Process. Mag., vol. 25, no. 2, pp. 21-30, Mar. 2008.

[15] A. Massa, P. Rocca, and G. Oliveri, "Compressive sensing in electromagnetics-a review," IEEE Antennas Propag. Mag., vol. 57, no. 1, pp. 224-238, Feb. 2015.

[16] X. Chen, "Subspace-based optimization method for solving inversescattering problems," IEEE Trans. Geosci. Remote Sens., vol. 48, no. 1, pp. 42-49, Jan. 2010

[17] M. Naeem, S. Xue, and D. Lee, "Cross-entropy optimization for sensor selection problems," in Proc. 9th Int. Symp. Commun. Inf. Technol., 2009, pp. 396-401.

[18] L. Yao, W. A. Sethares, and D. C. Kammer, "Sensor placement for on-orbit modal identification via a genetic algorithm," AIAA J., vol. 31, no. 10, pp. 1922-1928, 1993

[19] S. Lau, R. Eichardt, L. Di Rienzo, and J. Haueisen, "Tabu search optimization of magnetic sensor systems for magnetocardiography," IEEE Trans. Magn., vol. 44, no. 6, pp. 1442-1445, Jun. 2008.

[20] S. Joshi and S. Boyd, "Sensor selection via convex optimization," IEEE Trans. Signal Process., vol. 57, no. 2, pp. 451-462, Feb. 2009.

[21] P. Astrid, S. Weiland, K. Willcox, and T. Backx, "Missing point estimation in models described by proper orthogonal decomposition," IEEE Trans. Autom. Control, vol. 53, no. 10, pp. 2237-2251, Nov. 2008.

[22] K. Willcox, "Unsteady flow sensing and estimation via the gappy proper orthogonal decomposition," Comput. Fluids, vol. 35, no. 2, pp. 208-226, 2006.

[23] J. Ranieri, A. Chebira, and M. Vetterli, "Near-optimal sensor placement for linear inverse problems," IEEE Trans. Signal Process., vol. 62, no. 5, pp. 1135-1146, Mar. 2014.

[24] C. Jiang, Y. C. Soh, and H. Li, "Sensor placement by maximal projection on minimum eigenspace for linear inverse problems," IEEE Trans. Signal Process., vol. 64, no. 21, pp. 5595-5610, Nov. 2016.

[25] W. C. Chew, Waves and Fields in Inhomogeneous Media. Piscataway, NJ, USA: IEEE Press, 1995.
[26] J. Kong, Electromagnetic Wave Theory. New York, NY, USA: Wiley, 1986.

[27] G. Newsam and R. Barakat, "Essential dimension as a well-defined number of degrees of freedom of finite-convolution operators appearing in optics," J. Opt. Soc. Amer. A, vol. 2, no. 11, pp. 2040-2045, 1985.

[28] J. Xu and R. Janaswamy, "Electromagnetic degrees of freedom in 2-D scattering environments," IEEE Trans. Antennas Propag., vol. 54, no. 12, pp. 3882-3894, Dec. 2006.

[29] R. Solimene and R. Pierri, "Number of degrees of freedom of the radiated field over multiple bounded domains," Opt. Lett., vol. 32, no. 21 pp. 3113-3115, 2007.

[30] R. Piestun and D. A. Miller, "Electromagnetic degrees of freedom of an optical system," J. Opt. Soc. Amer. A, vol. 17, no. 5, pp. 892-902, 2000.

[31] V. Morozov and A. Grebennikov, Methods for Solution of Ill-Posed Problems: Algorithmic Aspects. Moscow, Russia: Univ. Press, 2005.

[32] Y. Sanghvi, Y. N. G. B. Kalepu, and U. Khankhoje, "Embedding deep learning in inverse scattering problems," IEEE Trans. Comput. Imag., vol. 6, pp. 46-56, 2019.

[33] X. Chen, Computational Methods for Electromagnetic Inverse Scattering. John Wiley \& Sons Singapore Pte. Ltd., 2018. [Online]. Available: https://onlinelibrary.wiley.com/doi/book/10.1002/9781119311997

[34] M. Grant and S. Boyd, "CVX: Matlab software for disciplined convex programming, version 2.1." Mar. 2014. [Online]. Available: https://cvxr. $\mathrm{com} / \mathrm{cvx}$

[35] M. Grant and S. Boyd, "Graph implementations for nonsmooth convex programs," in Recent Advances in Learning and Control, V. Blondel, S. Boyd, and H. Kimura, Eds. Lecture Notes in Control and Information Sciences, Berlin, Germany: Springer-Verlag, 2008, pp. 95-110. https:// stanford.edu/boyd/graph_dcp.html.

[36] A. Walther, "The question of phase retrieval in optics," Optica Acta: Int. J. Opt., vol. 10, no. 1, pp. 41-49, 1963.

[37] R. A. Gonsalves, "Phase retrieval and diversity in adaptive optics," Opt. Eng., vol. 21, no. 5, 1982, Art. no. 215829.

[38] R. P. Millane, "Phase retrieval in crystallography and optics," J. Opt. Soc. Amer. A, vol. 7, no. 3, pp. 394-411, 1990.

[39] D. Saldin, R. Harder, V. Shneerson, and W. Moritz, "Phase retrieval methods for surface x-ray diffraction," J. Phys.: Condens. Matter, vol. 13 no. 47, 2001, Art. no. 10689.

[40] J. R. Fienup, "Phase retrieval algorithms: A comparison," Appl. Optics, vol. 21, no. 15 , pp. $2758-2769,1982$.

[41] E. J. Candes, T. Strohmer, and V. Voroninski, "Phaselift: Exact and stable signal recovery from magnitude measurements via convex programming," Commun. Pure Appl. Math., vol. 66, no. 8, pp. 1241-1274, 2013.

[42] E. J. Candes, X. Li, and M. Soltanolkotabi, "Phase retrieval via wirtinger flow: Theory and algorithms," IEEE Trans. Inf. Theory, vol. 61, no. 4 pp. 1985-2007, Apr. 2015.

[43] K. Jaganathan, S. Oymak, and B. Hassibi, "Recovery of sparse 1-D signals from the magnitudes of their Fourier transform," in Proc. IEEE Int. Symp. Inf. Theory Proc., 2012, pp. 1473-1477.

[44] A. M. Tillmann, Y. C. Eldar, and J. Mairal, "Dolphin-dictionary learning for phase retrieval," IEEE Trans. Signal Process., vol. 64, no. 24, pp. 6485-6500, Dec. 2016.

[45] T. Qiu and D. P. Palomar, "Undersampled sparse phase retrieval via majorization-minimization," IEEE Trans. Signal Process., vol. 65, no. 22 pp. 5957-5969, Nov. 2017.

[46] O. M. Bucci, C. Gennarelli, and C. Savarese, "Representation of electromagnetic fields over arbitrary surfaces by a finite and nonredundant number of samples," IEEE Trans. Antennas Propag., vol. 46, no. 3, pp. 351-359, Mar. 1998 